\title{
Fluorescencia de la clorofila $a$ en plantas de piña sometidas a aplicación de herbicidas
}

\section{Fluorescence of chlorophyll a in pineapple plants submitted to herbicide applications}

JULIANO MIARI CORRÊA'

EVANDER ALVES FERREIRA ${ }^{1}$

GUSTAVO ANTÔNIO MENDES PEREIRA ${ }^{2,3}$

ALBA ROCIO AGUILAR PIRATOBA ${ }^{2}$

JOSÉ BARBOSA DOS SANTOS ${ }^{1}$

CARLOS HENRIQUE DE OLIVEIRA'

CÍCERO TEIXEIRA SILVA'

\section{Área experimental de plantación de piña.}

Foto: E.A. Ferreira

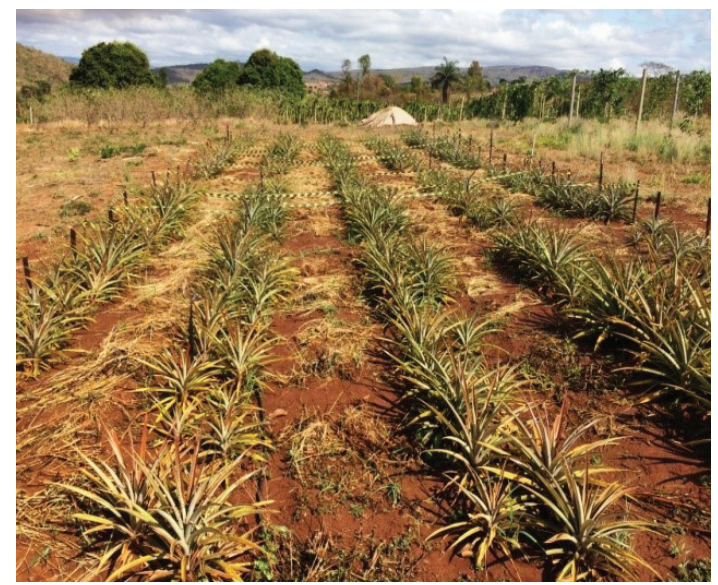

\section{RESUMEN}

El manejo químico de malezas con herbicidas es una actividad recomendada en cultivos, estrategia que permite un control eficaz para el cultivo de piña, que puede afectar su crecimiento y fisiología. De acuerdo a esto, el objetivo de este trabajo es evaluar el efecto de herbicidas de post-emergencia en parámetros de fluorescencia de la clorofila y tasa de transporte de electrones, relacionando la fluorescencia de la clorofila $a$ en plantas de piña, variedad Imperial, así como, comprobar la intoxicación causada por estos productos. El experimento se realizó en campo en bloques aleatorizados en esquema factorial $5 \times 2$, siendo cuatro herbicidas (ametym, diuron, fluazifop y sulfentrazona) y el control (sin herbicida) y dos tiempos de evaluación (7 y 21 días después de la aplicación). El monitoreo de la fluorescencia de clorofila y las tasas de transporte de electrones también se llevaron a cabo a los 7 y 21 días después de la aplicación del tratamiento, siendo las variables: 1) fluorescencia inicial; 2) fluorescencia máxima; 3) relación de fluorescencia variable y fluorescencia total y 4) tasa de transporte de electrones. Los herbicidas evaluados afectaron negativamente el aparato fotosintético de las plantas de piña, el herbicida diuron seguido del ametrym fueron los productos que provocaron mayor estrés fisiológico en el cultivo. En la segunda época de evaluación, los efectos sobre el aparato fotosintético de las plantas se fueron intensificando, donde los productos probados redujeron los valores de la relación fluorescencia variable y máxima, indicando aumento del estrés causado por los herbicidas evaluados.

Palabras clave adicionales: Ananas comosus, relación $\mathrm{F}_{\mathrm{v}} / \mathrm{F}_{\mathrm{m} \text { ’ }}$ ametrym, fluazifop-p-butyl.

1 Departamento de Agronomia, Universidade Federal dos Vales do Jequitinhonha e Mucuri, Diamantina-MG (Brasil). ORCID Corrêa, J.M.: 0000-0002-6592-7523; ORCID Ferreira, E.A.: 0000-0003-4701-6862; ORCID Santos, J.B.d.: 0000-0002-5746-7248; ORCID Oliveira, C.E.d.: 0000-0001-5130-7094; ORCID Silva, C.T.: 0000-0002-5268-3742

2 Departamento de Fitotecnia, Universidade Federal de Viçosa, Viçosa-MG (Brasil). ORCID Pereira, G.A.M.: 0000-0002-9063-3500; ORCID Piratoba, A.R.A.: 0000-0002-0939-3432

3 Autor para correspondencia. gustavogamp@hotmail.com 


\section{ABSTRACT}

The chemical management of weeds with herbicides is recommended for crops, a strategy that allows for the effective control of pineapple crops and that can affect growth and physiology. Accordingly, the objective of this study was to evaluate the effect of post-emergence herbicides on the fluorescence parameters chlorophyll and electron transport rate, relating chlorophyll a fluorescence in pineapple plants, Imperial variety, and checking for poisoning caused by these products. This experiment was carried out in the field in randomized blocks in a $5 \times 2$ factorial scheme, with four herbicides (ametym, diuron, fluazifop and sulfentrazone), a control (without herbicide) and two evaluation times (7 and 21 days after the application). The chlorophyll fluorescence monitoring and electron transport rates were carried out 7 and 21 days after the application of the treatment. The variables were: 1 ) initial fluorescence; 2) maximum fluorescence; 3) variable fluorescence ratio and total fluorescence; and 4) electron transport rate. The evaluated herbicides negatively affected the photosynthetic apparatus of the pineapple plants. The herbicide diuron, followed by ametrym, caused the greatest physiological stress in the crop. In the second evaluation period, the effects on the photosynthetic apparatus of the plants intensified, where the tested products reduced the values of the variables and maximum fluorescence ratio, indicating increased stress from the evaluated herbicides.

Additional key words: Ananas comosus, Fv/Fm ratio, ametrym, fluazifop-p-butyl.

Fecha de recepción: 15-08-2017 Aprobado para publicación: 30-01-2018

INTRODUCCIÓN

En el desarrollo milenario de la humanidad la fruticultura viene proporcionando los llamados alimentos funcionales (Moura et al., 2017; Costa et al., 2017). En la actualidad es una fuente de servicios y productos, siendo materia prima básica de diferentes sectores además del alimentario y desde la perspectiva financiera se puede considerar como un producto importante, mostrando su papel trascendental en la economía mundial, contribuyendo en la seguridad alimentaria y nutricional de la población humana (Bahiense et al., 2015).

Las regiones de la zona tórrida de la Tierra permiten una producción significativa de frutas durante todo el año, convirtiéndose en objetivo de trabajos investigativos y búsqueda de tecnologías apropiadas para reducir costos de producción, pero aumentando la productividad (Fachinello et al., 2011). Brasil se destaca por ser un importante productor de frutas de origen tropical y templado, cultivadas y nativas en sus diversos ecosistemas (MAPA, 2015).

La piña [Ananas comosus (L.) Merrill] es una de las frutas más tradicionales y consumidas en el mundo. Por sus cualidades y su aspecto imponente es apodado de "rey de las frutas". Originaria de Brasil, la piña tiene una variabilidad genética demostrada en más de 700 entradas presentes en el banco activo de germoplasma en la Unidad Descentralizada de la Empresa Brasileira de Pesquisa Agropecuária (EMBRAPA), subdivisión "EMBRAPA Mandioca y Fruticultura Tropical", en la ciudad de Cruz das Almas, en Bahía (Oliveira, 2017).

Brasil es el segundo país productor de piña, con una producción mundial del 15\%, equivalente a 1.470,3 mil toneladas y un área de plantación de 60,016 ha para el año 2010 (IBGE, 2016). Actualmente Filipinas es el mayor productor de piña y Costa Rica es el tercero (FAO, 2018).

Entre las principales variedades cultivadas la Imperial se destaca de otros genotipos, pues presenta resistencia a la fusariosis, enfermedad causada por el hongo Fusarium subglutinans, principal problema fitosanitario de esta planta en el país, generando pérdidas superiores al $80 \%$ de la producción. Es un híbrido resultante del cruce de 'Perolera' con 'Smooth Cayenne', obtenida por el programa de mejoramiento genético de piña de la EMBRAPA (2017). Produciendo frutos con pulpa de color amarillo, alto contenido de azúcar y excelente sabor en el análisis sensorial y tienen la ventaja de ausencia de espinas en las hojas (Oliveira, 2017). 
La interferencia negativa de malezas en cultivos de piña puede causar pérdidas en la productividad y en la calidad del fruto, siendo necesario adoptar alguna acción de control, que puede representar más de la mitad del costo de producción de la cosecha cuando es adoptado el control mecánico (Sripaoraya et al., 2011).

La competencia con malezas puede agravar el cultivo de piña pues al ser de aspecto menor y presentar un crecimiento vegetativo inicial muy lento, favorece la extracción de agua (especialmente en zonas influenciadas por períodos largos de sequía) y de nutrientes por parte de las malezas. La alta densidad (40 plantas/ $\mathrm{m}^{2}$ ) de coquito (Cyperus rotundus) y pasto herbáceo (Digitaria horizontalis) disminuye significativamente los contenidos de N, P, K y Ca en la hoja D de las plantas de piña, hoja reciente entre las hojas adultas y fisiológicamente más activa a los $30 \mathrm{~d}$ de competencia (Maia et al., 2012). La reducción de estos y otros nutrientes, interfieren en la productividad y la calidad de los frutos. Además, la morfología de la parte aérea del cultivo no permite el buen cierre del dosel facilitando la entrada de luz entre las hojas lo que permite el desarrollo de malezas, incluso en plantaciones densas (Cotunda et al., 2006).

El control químico con aplicación de herbicidas es una estrategia recomendada en el cultivo de piña, permitiendo un control efectivo, menor necesidad de mano de obra y reducción de costos de hasta un $80 \%$ si comparado al uso del control mecánico (Model et al., 2010). Los herbicidas más usados en cultivo de piña son los aplicados en pre-emergencia de malezas y de actividad residual relativamente larga en el suelo (Rodrigues y Almeida, 2011). Ese efecto es favorable para la piña por ser un cultivo de crecimiento lento y poca cobertura de la superficie del suelo (Maia et al., 2012). En algunos casos entretanto es común los flujos de emergencia de las malezas durante el período de crecimiento de la piña (Silva y Silva, 2013), situación que requiere aplicación de herbicidas en post-emergencia. Sin embargo, el número de herbicidas registrados para este modo de aplicación en cultivos de piña es menor y pueden causar daños al fruto en caso de herbicidas no selectivos, cuando las condiciones ambientales son desfavorables y/o cuando la tecnología de aplicación es inadecuada (Model et al., 2010).

Entre los herbicidas se pueden mencionar los que interfieren con la biosíntesis de la clorofila, resultando en la pérdida del pigmento, y en los cloroplastos alterándolos funcionalmente. Estos compuestos, además de interferir con la síntesis de clorofila, también puede afectar la formación de otros pigmentos, como las xantofilas y carotenoides, causando interferencia en el flujo de electrones y reducción en la producción de ATP por la inhibición de la actividad de la ATP- sintasa en el proceso de fotofosforilación (Silva y Silva, 2013).

Cuando hay un exceso de energía, puede disiparse en forma de fluorescencia (Taiz y Zeiger, 2017); por lo tanto, una forma de monitorear la inhibición o reducción en la transferencia de electrones entre los fotosistemas de la planta bajo tensión, que se puede observar aún en hojas intactas, es la fluorescencia de la clorofila en la que la reducción en la disipación de energía por el proceso fotoquímico es reflejada por el aumento correspondiente en la fluorescencia. El diurón y ametryn son inhibidores de la transferencia de electrones en el fotosistema II (PS II) que impediría la reducción de la quinona-A (Qa), al competir con la quinona-B $(\mathrm{Ob})$ por sitios de enlaces con la proteína $\mathrm{D}$, lo que provoca la salida de $\mathrm{Ob}$ y consecuentemente interrupción en el flujo de electrones, sin permitir la reducción del NADPH, que es esencial para la fijación de $\mathrm{CO}_{2}$ (Taiz y Zeiger, 2017). En consecuencia, hay reducción en la generación de energía y en las tasas fotosintéticas, resultando en aumento de la emisión de fluorescencia, además de causar la muerte de los tejidos verdes afectados por el producto.

Diferentes estudios han sido realizados utilizando la fluorescencia de la clorofila, para evaluar el efecto de herbicidas en la selectividad de plantas, como Catunda et al. (2005) en plantas de piña, Ferreira et al. (2015a) en plantas de mandioca y Ferreira et al. (2015b) en plantas de soja transgénica.

El control de malezas en cultivos de piña es una práctica indispensable, siendo cada vez más utilizado el control químico, que permite un control eficiente y no damnifica a las raíces del cultivo. Sin embargo, hay pocos estudios sobre la interferencia de herbicidas en el crecimiento del cultivo de interés.

Dado lo anterior, el objetivo de este estudio es evaluar el efecto de herbicidas de post-emergencia en parámetros relacionado a la fluorescencia de la clorofila $\boldsymbol{a}$ en plantas de piña (Ananas comusus), variedad Imperial, así como, evidenciar la intoxicación causada por estos productos en el cultivo.

\section{MATERIALES Y MÉTODOS}

Un ensayo de campo fue realizado en la Hacienda Experimental Río Manso, situada en Couto de 
Magalhães de Minas, Minas Gerais-BR (a 1807’ S y $43^{\circ} 47^{\prime}$ W; $726 \mathrm{~m}$ de altitud), la región presenta un índice pluviométrico anual medio de $1.269 \mathrm{~mm}$, temperatura anual media de $19,4^{\circ} \mathrm{C}$ donde plántulas de piña de la variedad Imperial fueron trasplantadas a campo, en el cual previamente fue recolectada muestras de suelos para análisis físico-químicas.

La clasificación del suelo utilizado corresponde a un Latosol Rojo Amarillo eutrófico profundo, de textura media con $45 \%$ de arena, $35 \%$ de arcilla, $20 \%$ de limo, densidad aparente de $1,63 \mathrm{~g} \mathrm{~cm}^{-3}$. De acuerdo con el análisis químico el suelo presento un $\mathrm{pH}$ de 5,77 y los valores de $\mathrm{P}$ y $\mathrm{K}\left(\mathrm{mg} \mathrm{dm}^{3}\right)$ de 5 y 84,31, respectivamente; Ca, $\mathrm{Mg} \mathrm{y} \mathrm{Al}^{+3}\left(\mathrm{cmol} \mathrm{dm}^{3}\right)$ de 1,64; 1,14 y 0,08 , respectivamente; $\mathrm{V} \%=53, \mathrm{MO}=0,38 \mathrm{dag} \mathrm{kg}^{-1}$.

Se plantaron en hoyos de $20 \times 20 \times 20 \mathrm{~cm}$ y una distancia entre las hileras dobles de 120 y $40 \mathrm{~cm}$ entre plantas colocadas alternativamente. Después de $30 \mathrm{~d}$ de adaptación de las plántulas, se aplicaron herbicidas de acuerdo a dosis comerciales como se describe en la tabla 1, esta aplicación fue realizada en el área total.

El diseño experimental fue en bloques al azar con cuatro repeticiones, con el esquema factorial $5 \times 2$, cuatro herbicidas (ametrym, diuron, sufentrazone y fluazifop) más un control (sin herbicida) y dos evaluaciones (7 y 21 d después de la aplicación del herbicida). Los datos se sometieron a análisis de varianza y las medias se compararon mediante la prueba de Tukey al $5 \%$ de probabilidad de error.

Evaluaciones visuales de intoxicación se llevaron a cabo a los 7 y 21 d después de la aplicación de los tratamientos (dat), en las plantas de piña adoptándose escala de porcentual, donde 0 significa que no hay síntomas, y $100 \%$ de muerte de la planta.

El monitoreo de la fluorescencia de la clorofila y la tasa de transporte de electrones (ETR) también se realizaron a los 7 y 21 dat en un tercio de la hoja D de las plantas de piña, utilizando el fluorómetro portátil de luz modulada MINI PAM ${ }^{\circledR}$. Las medidas de la fluorescencia de la clorofila fueron hechas después de 30 min de adaptación a la oscuridad, siendo realizadas las evaluaciones en el periodo nocturno, con emisión de pulsos de luz de 0,3 s, bajo frecuencia de $0,6 \mathrm{KHz}$. Las siguientes variables fueron evaluadas: 1) fluorescencia inicial $\left(F_{0}\right.$ - electrones quantum $\left.{ }^{-1}\right)$; 2) fluorescencia máxima $\left(F_{m}\right.$ - electrones quantum $\left.{ }^{-1}\right)$; 3) relación fluorescencia variable y máxima $\left(F_{v} / F_{m}\right)$ y 4) tasa de transporte de electrones (ETR - electrones $\mu$ mols $\mathrm{m}^{-2} \mathrm{~s}^{-1}$ ) (Ferreira et al., 2015a).

Los datos fueron tabulados y sometidos a análisis de varianza y al test de Tukey con una probabilidad del $5 \%$ de error.

\section{RESULTADOS Y DISCUSIÓN}

Las plantas de piña sometidas a la aplicación del herbicida diuron mostraron aumento de la fluorescencia inicial de la clorofila a $\left(\mathrm{F}_{0}\right)$ de la primera a la segunda época de evaluación, mientras que los otros tratamientos no presentaron diferencias entre las épocas con excepción del fluazifop, que a los 21 dat, las hojas seleccionadas para la lectura presentaron clorosis, evolucionando a necrosis y finalmente a la muerte de los tejidos. Al evaluar el efecto de los herbicidas dentro de cada época de evaluación, se encontró que a los 7 dat, las plantas tratadas mostraron mayor valor medio de $\mathrm{F}_{0}$ del ametryn diferente a los otros tratamientos. Después de $21 \mathrm{~d}$ los mayores valores de $\mathrm{F}_{0}$ se observaron en las plantas tratadas con ametrym y diuron (Tab. 2).

Valores mayores de $\mathrm{F}_{0}$ pueden indicar deterioros estructurales directos en los centros de reacción o efecto indirecto de los mismos en el fotosistema II comprometiendo el transporte de energía de excitación de los complejos antena para los centros de reacción (Ferreira et al., 2015a). Según los mismos autores, la $F_{0}$ no

Tabla 1. Productos comerciales probados en plantas de piña (variedad Imperial).

\begin{tabular}{|l|c|c|c|}
\hline Marca comercial (®) & Ingrediente activo (i.a.) & $800 \mathrm{~g} \mathrm{~kg}^{-1}$ & $3,0 \mathrm{~kg} \mathrm{ha}^{-1}$ \\
\hline Ametrex WG & Ametryn & $800 \mathrm{~g} \mathrm{~kg}^{-1}$ & $3,0 \mathrm{~L} \mathrm{ha}^{-1}$ \\
\hline Diuron Nortox & Diuron & $250 \mathrm{~g} \mathrm{~L}^{-1}$ & $1,0 \mathrm{~L} \mathrm{ha}^{-1}$ \\
\hline Fusilade & Fluazifop-p-butyl & $500 \mathrm{~g} \mathrm{~L}^{-1}$ & $1,2 \mathrm{~L} \mathrm{ha}^{-1}$ \\
\hline Boral 500 & Sulfentrazone & \\
\hline
\end{tabular}

${ }^{1}$ Dosis recomendada (Agrofit - MAPA, 2015); 2Producto no registrado para cultivo de piña. 
Tabla 2. Fluorescencia inicial $\left(F_{0}\right)$ electrones quantum-1) en plantas de la piña sometidas a la aplicación de herbicidas.

\begin{tabular}{|l|c|c|}
\hline \multirow{2}{*}{\multicolumn{1}{|c|}{ Herbicidas }} & \multicolumn{2}{|c|}{ Épocas } \\
\cline { 2 - 3 } & $5 \mathrm{~d}$ & $21 \mathrm{~d}$ \\
\hline Ametryn & $57,30 \mathrm{aA}$ & $55,35 \mathrm{aA}$ \\
\hline Diuron & $52,50 \mathrm{bB}$ & $58,50 \mathrm{aA}$ \\
\hline Sulfentrazone & $49,10 \mathrm{aB}$ & $49,90 \mathrm{aB}$ \\
\hline Fluazifop & $52,25 \mathrm{aB}$ & $00,00 \mathrm{bC}$ \\
\hline Control & $49,80 \mathrm{aB}$ & $46,50 \mathrm{aB}$ \\
\hline CV (\%) & \multicolumn{2}{|c|}{16,11} \\
\hline
\end{tabular}

Promedios con letras distintas en minúscula (renglón) y mayúscula (columna) indican diferencia significativa según la prueba de Tukey $(P \leq 0,05)$.

siempre es una constante, su valor puede aumentar si los centros de reacción de PSII están comprometidos, o si la transferencia de la energía de excitación de la antena para los centros de reacción este perjudicada. Se destaca que los herbicidas ametryn y diuron afectan directamente el transporte de electrones enlazándose a la proteína D1 e interrumpiendo la fotosíntesis, por lo que la planta tiende a liberar energía de otra manera como fluorescencia y calor (Tropaldi et al., 2015; Silva y Silva, 2013; Taiz y Zeiger, 2017).

En la fase de transporte de electrones durante la fotosíntesis en las plantas, la luz es absorbida por complejos de pigmentos de antena, que al excitar los electrones transfieren energía a los centros de reacción de los fotosistemas II y I (Taiz y Zeiger, 2017). Cuando hay un exceso de energía, esta puede ser disipada en forma de fluorescencia. Por lo tanto, una forma de monitoreo de la inhibición o reducción en la transferencia de electrones entre los fotosistemas de las plantas bajo la aplicación de herbicidas es la fluorescencia de la clorofila (Taiz y Ziger, 2017), que puede ser observada aun en hojas intactas (Ferreira et al., 2015a; Paula et al., 2015; Taiz y Zeiger, 2017), en que la reducción en la disipación de la energía por el proceso fotoquímico es reflejada por un aumento correspondiente en la fluorescencia.

Al evaluar la fluorescencia máxima de la clorofila a $\left(\mathrm{F}_{\mathrm{m}}\right)$ en plantas de piña, se verificó que el herbicida sulfentrazone mostró mayor valor medio de esa variable a los 7 dat diferenciándose de la segunda época de evaluación (a los 21 dat) para los otros tratamientos no hubo diferencias entre las épocas de evaluación. Al evaluar el efecto de los herbicidas dentro de cada época, se observó que el ametrym promovió el menor valor de $\mathrm{F}_{\mathrm{m}}$ en relación a los demás tratamientos a los 7 dat, a los 21 dat se verificó valores mayores de $\mathrm{F}_{\mathrm{m}}$ en las plantas de piña tratadas con ametrym y diuron. De otro lado el fluazifop inhibió toda la actividad en las hojas (Tab. 3).

Tabla 3. Fluorescencia máxima $\left(\mathrm{F}_{\mathrm{m}}\right.$ - electrones quantum $\left.{ }^{-1}\right)$ en plantas de piña sometidas a la aplicación de herbicidas.

\begin{tabular}{|l|c|c|}
\hline \multirow{2}{*}{\multicolumn{1}{|c|}{ Herbicidas }} & \multicolumn{2}{|c|}{ Épocas } \\
\cline { 2 - 3 } & $\mathbf{d}$ & $21 \mathrm{~d}$ \\
\hline Ametryn & $185,00 \mathrm{aB}$ & $181,67 \mathrm{aA}$ \\
\hline Diuron & $223,45 \mathrm{aA}$ & $194,20 \mathrm{aA}$ \\
\hline Sulfentrazone & $213,35 \mathrm{aA}$ & $163,50 \mathrm{bB}$ \\
\hline Fluazifop & $226,00 \mathrm{aA}$ & $00,00 \mathrm{bC}$ \\
\hline Control & $226,80 \mathrm{aA}$ & $224,80 \mathrm{aA}$ \\
\hline CV (\%) & \multicolumn{2}{|c|}{17,1} \\
\hline
\end{tabular}

Promedios con letras distintas en minúscula (renglón) y mayúscula (columna) indican diferencia significativa según la prueba de Tukey $(P \leq 0,05)$.

La relación de fluorescencia variable y máxima $\left(\mathrm{F}_{\mathrm{v}}\right)$ $\mathrm{F}_{\mathrm{m}}$ ) varió en las épocas de aplicación de los herbicidas utilizados, excepto la del control. A los 7 dat sólo las parcelas tratadas con ametrym mostraron reducción en los valores de $\mathrm{F}_{\mathrm{v}} / \mathrm{F}_{\mathrm{m}}$ por debajo del 0,75. Las plantas tratadas con herbicidas mostraron reducción en los valores $\mathrm{F}_{\mathrm{v}} / \mathrm{F}_{\mathrm{m}}$ a los 21 dat, sin embargo, esos valores observados se encuentran fuera del intervalo ideal para todos los productos probados, lo que indica que la planta de piña estaba sobre el estrés (Tab. 4).

Es importante destacar que este intervalo ideal corresponde los valores ingresados entre 0,75 (límite inferior) y 0,85 (máximo). El rendimiento cuántico máximo de PS II $\left(\mathrm{F}_{\mathrm{v}} / \mathrm{F}_{\mathrm{m}}\right)$ puede variar de 0.75 a 0,85 en plantas no sometidas a estrés (Corrêa y Alves, 2010), siendo la reducción de esta relación un excelente indicador del efecto fotoinhibidor cuando las plantas se someten a estrés químico (Jiménez-Suancha et al., 2015).

Las plantas de piña tratadas con los herbicidas diuron y sulfentrazone mostraron reducción de $\mathrm{F}_{\mathrm{v}} / \mathrm{F}_{\mathrm{m}}$ entre la primera y la segunda evaluación, se resalta que la $F_{v} /$ $\mathrm{F}_{\mathrm{m}}$ en la primera época estaba dentro del límite ideal $(0,75$ a 0,85$)$, ya para la segunda época de evaluación los valores de $\mathrm{F}_{\mathrm{v}} / \mathrm{F}_{\mathrm{m}}$ estaban por fuera del límite ideal (Tab. 4). Otra forma de interpretar está relacionada a valores estándar de una planta sin deterioro al aparato fotosintético, que están alrededor de $0,80 \mathrm{~F}_{\mathrm{v}} / \mathrm{F}_{\mathrm{m}}$, 
y cuando las plantas exhiben un valor por debajo de este se considera que la maquinaria fotosintética fue perjudicada (Chaves, 2015). No hubo evaluación en las parcelas del herbicida fluazifop, pues a los $21 \mathrm{~d}$, las hojas estaban muertas.

Al evaluar el efecto de los tratamientos con herbicidas dentro de cada época, se verificó que en la primera época el herbicida ametrym afecto negativamente a $\mathrm{F}_{\mathrm{v}} / \mathrm{F}_{\mathrm{m}}$ diferenciándose de los otros herbicidas y el control, sin embargo, a los 21 dat se observó que estos afectaron negativamente a $\mathrm{F}_{\mathrm{v}} / \mathrm{F}_{\mathrm{m}}$, donde los valores observados estaban fuera del límite ideal de $\mathrm{F}_{\mathrm{v}} / \mathrm{F}_{\mathrm{m}}$, indicando estrés en el aparato fotosintético causado por todos los productos ensayados (Tab. 4).

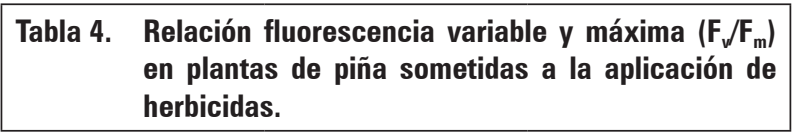

\begin{tabular}{|l|c|c|}
\hline \multirow{2}{*}{\multicolumn{1}{c|}{ Herbicidas }} & \multicolumn{2}{c|}{ Épocas } \\
\cline { 2 - 3 } & 7 días & 21 días \\
\hline Ametryn & $0,655 \mathrm{bB}$ & $0,722 \mathrm{aAB}$ \\
\hline Diuron & $0,765 \mathrm{aA}$ & $0,690 \mathrm{bB}$ \\
\hline Sulfentrazone & $0,755 \mathrm{aA}$ & $0,672 \mathrm{bB}$ \\
\hline Fluazifop & $0,760 \mathrm{aA}$ & $0,00 \mathrm{bC}$ \\
\hline Control & $0,772 \mathrm{aA}$ & $0,757 \mathrm{aA}$ \\
\hline CV (\%) & \multicolumn{2}{|c|}{4,65} \\
\hline
\end{tabular}

Promedios con letras distintas en minúscula (renglón) y mayúscula (columna) indican diferencia significativa según la prueba de Tukey $(P \leq 0,05)$.

Tanto en la presencia del herbicida ametrym como del diuron, el sistema de protección fotosintético dado por los carotenoides, es rebasado por un exceso de clorofila en el estado de energía triple, debido a que las moléculas del herbicida se enlazan a la proteína D1 del PS II, interrumpiendo el flujo electrones (Paiva et al., 2015), de esta forma, la clorofila con una carga triple, en presencia de oxígeno conduce a la peroxidación de membranas del cloroplasto, debido a la formación de peróxido de hidrógeno, lo que explica la aparición de clorosis en las hojas, pasando por la necrosis, a medida que el proceso alcanza la pared celular (Silva y Silva, 2013).

El sulfentrazone así como el ametrym y el diuron tienen acción directa sobre el aparato fotosintético (Torres et al., 2012), sin embargo, el sulfentrazone funciona inhibiendo la síntesis de la clorofila, actúa en la enzima PROTOX en la ruta de síntesis de la clorofila promoviendo la producción de radicales libres altamente reactivos en el interior celular (Silva y Silva, 2013; Ferreira et al., 2012).
El fluazifop-P-butil, tiene como objetivo principal el control de monocotiledóneas (Cesarin et al., 2013; Fontes et al., 2013; Rezende et al., 2012). El herbicida fluazifop-P-butil actúa inhibiendo la enzima acetil- CoA carboxilasa (ACC), bloqueando la síntesis de lípidos en las plantas susceptibles (Pereira et al., 2015; Silva et al., 2014), de esta forma su acción en el aparato fotosintético es indirecto.

Las plantas de piña sometidas a aplicación de los herbicidas diuron y sulfentrazone mostraron reducción en la tasa de transporte de electrones (ETR) de la primera a la segunda época de evaluación, los otros tratamientos no presentaron diferencias significativas entre las épocas, siendo que no hubo evaluación para el herbicida fluazifop porque las plantas a los $21 \mathrm{~d}$ después de la aplicación tenían las hojas inertes. Al evaluar el efecto de los herbicidas dentro de cada época de evaluación, se constató que a los 7 dat las plantas tratadas con ametrym mostraron menor valor medio de ETR diferente de los demás tratamientos, con relación a la segunda época de evaluación no hubo diferencias entre los tratamientos (Tab. 5).

\section{Tabla 5. Tasa de transporte de electrones (ETR - $\mu$ mols electrones $\mathrm{m}^{-2} \mathrm{~s}^{-1}$ ) en plantas de piña sometidas a la aplicación de herbicidas en piña.}

\begin{tabular}{|l|c|c|}
\hline \multirow{2}{*}{\multicolumn{1}{|c|}{ Herbicidas }} & \multicolumn{2}{|c|}{ Épocas } \\
\cline { 2 - 3 } & 7 días & 21 días \\
\hline Ametryn & $12,51 \mathrm{aB}$ & $13,77 \mathrm{aA}$ \\
\hline Diuron & $15,44 \mathrm{aAB}$ & $12,22 \mathrm{bA}$ \\
\hline Sulfentrazone & $16,53 \mathrm{aAB}$ & $12,55 \mathrm{bA}$ \\
\hline Fluazifop & $16,85 \mathrm{aA}$ & $0,00 \mathrm{bB}$ \\
\hline Control & $14,61 \mathrm{aA}$ & $13,11 \mathrm{aA}$ \\
\hline CV (\%) & \multicolumn{2}{|c|}{13,10} \\
\hline
\end{tabular}

Promedios con letras distintas en minúscula (renglón) y mayúscula (columna) indican diferencia significativa según la prueba de Tukey $(P \leq 0,05)$.

En el trabajo, llevado a cabo por Dayan et al. (2009), fue monitoreado el ETR en plantas de Digitaria sanguinalis y Abutilon theophrasti, sometidas a la aplicación de atrazine y amicarbazone. La tasa de transporte de electrones para ambas especies fue inhibida completamente ocho horas después de la aplicación de los herbicidas.

Araldi et al. (2012), evaluando el efecto del haloxyfop-methyl en la ETR en plantas de $U$. decumbens, verificaron que con la aplicación de herbicidas en $U$. decumbens, las plantas mostraron pleno funcionamiento, con $100 \%$ del ETR del control en hasta 24 
h después de la aplicación; sin embargo, a partir de 72 h la reducción del ETR fue del $42 \%$, siendo que, en intervalo de 120-312 h, el ETR se mantuvo en el rango de $50 \%$ de reducción. A las 318 h después de la aplicación la reducción del ETR pasó a $65 \%$, y con 432 $\mathrm{h}$ las plantas mostraron una completa inhibición del transporte de electrones.

La piña es una planta que pertenece al grupo de metabolismo ácido de las crasuláceas CAM (Aragón et al., 2012; Weise et al., 2011). Además, existe una divergencia en el metabolismo de esta planta, clasificándola en una planta CAM facultativa, cuando las plantas se encuentran en ambientes de menor radiación y baja temperatura; con buena disponibilidad hídrica ellas se comportarían como C3, y cuando expuestas a luminosidad excesiva, déficit hídrico, o altas temperaturas que tornan la absorción diurna del $\mathrm{CO}_{2}$ menos favorable, esas plantas pasan a presentar el comportamiento CAM (Luttge, 2004; Taybi et al., 2002).

\section{CONCLUSIONES}

Los herbicidas evaluados afectan negativamente el aparato fotosintético de las plantas de piña, alterando la fluorescencia inicial, fluorescencia máxima, relación de fluorescencia variable y máxima, fluorescencia total y tasa de transporte de electrones, siendo que el ametrym y el diuron son los más perjudiciales. Con el tiempo los efectos en el aparato fotosintético de las plantas son intensificados, donde los productos ensayados reducen los valores de la relación de fluorescencia variable y máxima, indicando aumento del estrés causado por los herbicidas evaluados.

\section{AGRADECIMIENTOS}

Los autores expresan sus agradecimientos a las agencias brasileñas: Conselho Nacional de Desenvolvimento Científico y Tecnológico (CNPq), Coordenação de Aperfeiçoamento de Pessoal de Nível Superior (CAPES) y Fundação de Amparo à Pesquisa do Estado de Minas Gerais (FAPEMIG), por las becas de estudio y ayudas concedidas.

Conflicto de intereses: el manuscrito fue preparado y revisado con la participación de los autores, quienes declaran no tener algún conflicto de interés que coloquen en riesgo la validez de los resultados aquí presentados.

\section{REFERENCIAS BIBLIOGRÁFICAS}

Aragón, C., L. Carvalho, J. González, M. Escalona, M. y S. Amancio. 2012. The physiolgy of ex vitro pineapple (Ananas comosus L. Merril var MD-2) as CAM or C3 is regulated by environmental conditions. Plant Cell Rep. 31(4), 757-769. Doi: 1007/s00299-011-1195-7

Araldi, R., M. Girotto, E.D. Velini, G.L.G.C. Gomes, S.P. Jasper, C.A. Carbonari y M.L.B. Trindade. 2012. Eficiência fotossintética e consumo de água de Ipomoea triloba após aplicação de herbicidas. Planta Daninha 30(3), 517-524. Doi: 10.1590/S0100-83582012000300007

Bahiense, D.V., P.M. Souza y N.J. Ponciano. 2015. Incentivos à produção de frutas e as mudanças na agricultura da região norte do estado do Rio de Janeiro. Rev. Bras. Frutic. 37(2), 387-395. Doi: 10.1590/0100-2945-096/14

Catunda, M.G., S.P. Freitas, J.G. Oliveira y C.M.M. Silva. 2005. Efeitos de herbicidas na atividade fotossintética e no crescimento de abacaxi (Ananas comossus). Planta Daninha 23(1), 115-121. Doi: 10.1590/ S1413-70542010000500009

Cesarin, A.E., J.F. Martins, P.R.F. Giancotti, M.P. Nepumuceno y P.L.C.A. Alves. 2013. Eficácia de herbicidas no controle pós-emergente de Sisyrinchium micranthum Cav. e Agrostis sp. Rev. Bras. Herb. 12(3), 296-306. Doi: 10.7824/rbh.v12i3.216

Chaves, V.V. 2015. Trocas gasosas e fluorescência da clorofila "a" em genótipos de cana-de-açúcar infestados por cigarrinha-das-raízes Mahanarva fimbriolata. Dissertação de Mestrado. Departamento de Fitotecnia, Universidade Federal de Viçosa

Corrêa, M.J.P. y P.L.C.A. Alves. 2010. Efeitos da aplicação de herbicidas sobre a eficiência fotoquímica em plantas de soja convencional e geneticamente modificada. Ciênc. Agrotec. 34(5), 1136-1145. Doi: 10.1590/ S1413-70542010000500009

Costa, A.C., J.D. Ramos, T.P. Menezes, R.R. Laredo y M.H. Duarte. 2017. Quality of pitaia fruits submitted to field bagging. Rev. Bras. Frutic. 39(Spe.), 1-5. Doi: 10.1590/0100-29452017377

Dayan, F.E., M.L.B. Trindade y E.D. Velini. 2009. Amicarbazone, a new photosystem II inhibitor. Weed Science 57(6), 579-583. Doi: 10.1614/WS-09-099.1

EMBRAPA. 2017. Adubação para alta produtividade e qualidade: Fruteiras tropicais do Brasil. Boletim Embrapa. Doi: 10.3235/978-3-9523243-1-8

Fachinello, J.C., M.S. Pasa, J.D. Schmtiz y D.L. Betemps. 2011. Situação e perspectivas da fruticultura de clima temperado no Brasil. Rev. Bras. Frutic. (Esp.), 109-120. Doi: 10.1590/S0100-29452011000500014. 
FAO (Food and Agriculture Organization of the United Nations). 2018. FAOSTAT Countries by commodity. Pineapples. FAO, Roma.

Ferreira, E.A., C.C. Matos, E.A. Barbosa, D.V. Silva, J.B. Santos, G.A.M. Pereira, A.T. Faria y C.T. Silva. 2015a. Respostas fisiológicas da mandioca à aplicação de herbicidas. Semina Ciênc. Agrár. 36(2), 645-656. Doi: 10.5433/1679-0359.2015v36n2p645.

Ferreira, E.A., C.C. Matos, E.A. Barbosa, C.A.D. Melo, D.V. Silva y J.B. Santos. 2015b. Aspectos fisiológicos de soja transgênica submetida à competição com plantas daninhas. Rev. Ciênc. Agrár. 58(2), 115-121. Doi: 10.4322/rca.1745.

Ferreira, E.A., A.F. Silva, A.A. Silva, D.V. Silva, L.L. Galon y J.B. Santos. 2012. Toxidade de herbicidas a genótipos de cana-de-açúcar. Rev. Tróp. Ciênc. Agrár. Biol. 6(1), 84-92.

Fontes, J.R., M.V. Garcia, y R.N. Rocha. 2013. Seletividade de Herbicidas para o abacaxizeiro. En: Anais do V Simpósio brasileiro da cultura do abacaxi. Secretaria da Agricultura e Pecuária do Estado de Tocantins. Palmas-TO, Brasil.

Fontes, J.R.A., I.J. Oliveira y J.R.P. Gonçalves. 2013. Seletividade e eficácia de herbicidas para cultura do feijãocaupi. Rev. Bras. Herb. 12(1), 47-55. Doi: 10.7824/rbh. v12i1.214.

IBGE - Instituto Brasileiro de Geografía e Estatística. 2016. LSPA. En: ftp://ftp.ibge.gov.br/ Producao_Agricola/Levantamento_Sistematico_ da_Producao_Agricola_[mensal]/Fasciculo/2016/ 1spa_201612_20170222_133000.pdf; consultado: junio de 2017.

Jiménez-Suancha, S.C., S.O.H. Alvarado y H.H. Balaguera-López. 2015. Fluorescencia como indicador de estrés en Helianthus annuus L. Una revisión. Rev. Colomb. Cienc. Hortíc. 9(1), 149-160. Doi: 10.17584/ rcch.2015v9i1.3753.

Luttge, U. 2004. Ecophysiology of crassulacean acid metabolism (CAM). Ann. Bot. 93(6), 629-652. Doi: 10.1093/aob/mch087.

Maia, L.C.B., V.M. Maia, M.H.M. Lima, I. Aspiazú y R.F. Pegoraro. 2012. Growth, production and quality of pineapple in response to herbicide use. Rev. Bras. Frutic. 34(3), 799-805. Doi: 10.1590/ S0100-29452012000300020.

MAPA. 2015. Produção integrada no Brasil: agropecuária sustentável alimentos seguros. Ministério da Agricultura, Pecuária e Abastecimento. Secretária de Desenvolvimento Agropecuário e Cooperativismo. Brasília: Mapa/ACS. En: http://www.agricultura.gov. br; consultado: junio de 2017.

Model, N.S., R. Favreto y A.E.C. Rodrigues. 2010. Efeitos de tratamentos de controle de plantas daninhas sobre produtividade, sanidade e qualidade do abacaxi. Pesq. Agropecu. Gaúcha 16(1), 51-58.
Moura, G.C.M., M. Vizzotto, L. Picolotto y L.E.C. Antunes. 2017. Production, physical-chemical quality and bioative compounds of misty blueberry fruit under different pruning intensities. Rev. Bras. Frutic. 39(Spe), 1-6. Doi: 10.1590/0100-29452016158.

Oliveira, C.H. 2015. Ação de herbicidas sobre o abacaxizeiro micropropagado e micorrizado. Dissertação de mestrado. Programa de Pós-Graduação em Produção Vegetal. Universidade Federal dos Vales do Jequitinhonha e Mucuri, Diamantina-MG, Brasil.

Paiva, M.C.G., L.H. Barcellos Jr., G.A.M. Pereira, V.A. Gonçalves, R.S. Felipe, M.S. Machado y A.A. Silva. 2015. Fitotoxicidade de herbicidas aplicados em pós transplantio de mudas de maracujá amarelo. Rev. Bras. Frutic. 14(4), 280-287. Doi: 10.7824/rbh.v14i4.446.

Paula, S.M., E.R. Naves, I.M. Franco, K.J.M. Padua, K.R. Silva, W.P. Fernandes, D.C. Oliveira, J.P. Lemos-Filho y A.S.F.P. Moreira. 2015. Desempenho fotossintético de folhas jovens e maduras de Vochysia cinnamomea (Vochysiaceae) em áreas de cerrado rupestre intactas e pós-queimada. Biosci. J. 31(2), 591-600. Doi: 10.14393/ BJ-v31n2a2015-23474.

Pereira, G.A.M., L.H. Barcellos Jr., D.V. Silva, R.R. Braga, A.A. Silva, M.M. Teixeira y J.L. Ribeiro Jr. 2015. Application height in herbicides efficiency in bean crops. Planta Daninha 33(3), 607-614. Doi: 10.1590/ S0100-83582015000300023.

Rezende, B.P.M., C.J. Tavares, R.E. Marangoni, P.C.R. Cunha y A. Jakelaitis. 2012. Efeito do fomesafen + fluazifop-p-butil associados com inseticidas no controle das plantas daninhas na cultura da soja. Rev. Bras. Ciênc. Agrár. 7(4), 608-613. Doi: 10.5039/agraria. v7i4a1842.

Rodrigues, B.N. y F.S. Almeida. 2011. Guia de herbicidas. $6^{a}$ ed. Edição dos autores, Londrina-PR, Brasil.

Silva, A.A., J.F. Silva. 2013. Tópicos em manejo de plantas daninhas. Universidade Federal de Viçosa,Viçosa-MG, Brasil.

Silva, D.V., H.M. Silveira, E.A. Ferreira, F.P. Carvalho, M.D. Castro Neto, A.A. Silva y T. Sediyama. 2014. Aspectos fisiológicos da mandioca após a aplicação dos herbicidas fluazifop-p-butil e fomesafen. Rev. Ceres 61(2), 178-183. Doi: 10.1590/S0034-737X2014000200004.

Sripaoraya, S., M.R. Davey y P. Srinives. 2011. F1 hybrid resistant pineapple to bialaphos herbicide. Acta Hortic. 902(1), 201-207. Doi: 10.17660/Acta Hortic.2011.902.20.

Taiz, L. y E. Zeiger. 2017. Fisiologia vegetal. Artmed, Porto Alegre-RS, Brasil.

Taybi, T., J.C. Cushman y A.M. Borland. 2002. Environmental, hormonal and circadian regulation of crassulacean acid metabolism expression. Functional Plant Biol. 29(6), 669-678. Doi: 10.1071/PP01244. 
Torres, L.G., E.A. Ferreira, P.R.R. Rocha, A.T. Faria, V.A. Gonçalves, L. Galon, A.F. Silva y A.A. Silva. 2012. Alterações nas características fisiológicas de cultivares de cana-de-açúcar submetida à aplicação de herbicidas. Planta Daninha 30(3), 581-587. Doi: 10.1590/ S0100-83582012000300014.

Tropaldi, L., E.D.Velini, C.A. Carbonari, R. Araldi, N. Corniani, M. Girotto y I.P.F. Silva. 2015. Detecção da tolerância de diferentes espécies de capim-colchão a herbicidas inibidores do fotossistema II utilizando a técnica da fluorescência. Ciênc. Rural 45(5), 767-773. Doi: 10.1590/0103-8478cr20140506.

Weise, S.E., K.J.V. Wijk y T.D. Sharkey. 2011. The role of transitory starch in C3, CAM, and C4 metabolism and opportunities for engineering leaf starch accumulation. J. Exp. Bot. 62(9), p 3109-3118. Doi: 10.1093/ jxb/err035. 